\title{
Evaluating a Novel Hemodialysis Central Venous Catheter Cap in Reducing Bloodstream Infections: A Quality Improvement Initiative
}

\author{
Steven Weiss' \\ Muhammad Qureshi ${ }^{2}$ \\ 'Quality and Support Services, Atlantic \\ Dialysis Management Services, \\ Ridgewood, New York, USA; ${ }^{2}$ Clinical \\ Research, Mattoo \& Bhat Medical \\ Associates, P.C., College Point, \\ New York, USA
}

Correspondence: Steven Weiss Quality and Support Services, Atlantic Dialysis Management Services, 385 Seneca Ave, Ridgewood, NY, II 385 , USA

Tel + I 347-5/2-9090

$\mathrm{Fax}+1$ 7|8-366-2936

Email sweiss@atlanticdialysis.com
Purpose: Central line-associated bloodstream infection (CLABSI) is the second leading cause of death in hemodialysis patients. Patients dialyzed via central venous catheters (CVCs) are more susceptible to vascular access-related bloodstream infection (VRBSI), sepsis, and mortality when compared to patients with other dialysis accesses.

Patients and Methods: A retrospective observational data analysis was conducted from 13 outpatient dialysis clinics in the United States to compare novel chlorhexidine-coated end caps to standard needlefree connectors for differences in CLABSI rates when utilizing CVCs for hemodialysis. There were two periods in this study: in the first study period over a 5-month period (May 2018 to September 2018), data were evaluated from a group of patients undergoing hemodialysis using chlorhexidine end-caps ('chlorhexidine group') as well as a group using standard needlefree connectors ('standard group'). An initial assessment found that a substantial CLABSI rate reduction was seen with use of chlorhexidinecoated end caps; therefore, most patients were switched to chlorhexidine by February 2019 and data continued to be collected till June 2019. The second study period spanned 9 months from October 2018 to June 2019.

Results: Across 13 dialysis centers, anonymized health records of 5934 patients who were dialyzed via CVCs between May 2018 and June 2019 were analyzed. The mean age was 61.3 and $47.1 \%$ of all patients were female. Study period one included 967 patients with chlorhexidine and 1044 patients with standard end caps, while there were 3647 chlorhexidine and 276 standard patients in the second period. The combined CLABSI rate in the chlorhexidine group was 0.09 / 1000 CVC days versus $0.63 / 1000 \mathrm{CVC}$ days in the standard group $(\mathrm{p}<0.0001)$.

Conclusion: Chlorhexidine-coated $\mathrm{CVC}$ caps may provide a therapeutic improvement in CVC hemodialysis management.

Keywords: catheter-related infection, renal dialysis, quality improvement, infection control, chronic kidney failure, chlorhexidine-coated CVC caps

\section{Introduction}

A bloodstream infection (BSI) is the presence of one or more viable pathogens in systemic circulation confirmed by positive microbiological blood culture(s). BSIs can elicit a lifethreatening systemic inflammatory response characterized by fever, chills and/or hypotension. Indwelling devices, namely central venous catheters (CVCs), are common sources of secondary BSIs in outpatient hemodialysis settings where vascular access is essential in carrying out a hemodialysis procedure. ${ }^{1,2}$ In fact, central line-associated bloodstream infections (CLABSIs) are the second leading cause of death in hemodialysis patients, with an attributable mortality rate ranging between $12 \%$ and $25 \%$ and estimated 
costs of 45,000 USD per event. ${ }^{3,4}$ Approximately 500,000 patients receive hemodialysis for end-stage renal disease annually in the United States, and are susceptible to BSIs arising from frequent hospitalizations and weakened immune systems. ${ }^{3,5}$ Over $80 \%$ of these patients initiate hemodialysis via CVCs, a modality associated with a higher risk of vascular access-related bloodstream infection (VRBSI), sepsis, and mortality compared to patients dialyzed via arteriovenous fistulas (AVFs) or arteriovenous grafts (AVGs). ${ }^{6}$ According to the Center for Disease Control's National Healthcare Safety Network (NHSN), $69.8 \%$ of VRBSIs in the United States can be attributed to CVCs. ${ }^{5}$ Specifically, the CLABSI rate per thousand CVC days is reported as 0.71 for CVCs, which is significantly higher than 0.09 for AVFs, and 0.13 for AVGs. ${ }^{5}$

Recent advances in CVC technology such as needleless connectors and disinfecting caps have been identified as effective strategies to reduce CLABSI incidence. ${ }^{7}$ National Kidney Foundation's Kidney Disease Outcomes Quality Initiative (KDOQI) has also emphasized the importance of preventing CVC dysfunction in the latest Clinical Practice Guidelines for Vascular Access, and recommends the use of antimicrobial barrier caps to help reduce catheter-related bloodstream infections in high-risk patients or facilities. ${ }^{8}$ To this notion, a novel CVC cap with antimicrobial coated rods featuring the broadspectrum biocide chlorhexidine, has demonstrated lower rates for CLABSI and positive blood cultures in CVC hemodialysis patients in two randomized clinical trials. ${ }^{9,10}$ Herein, findings are reported from a multi-center quality improvement assessment conducted at a US-based outpatient dialysis network to evaluate CLABSI rates following the use of these novel chlorhexidine-coated CVC end caps.

\section{Patients and Methods}

\section{Project Design and Setting}

This study is a retrospective analysis of data collected from a quality improvement assessment conducted at 13 outpatient dialysis clinics across New York, USA (New York City and Long Island), all of which are members of a regional dialysis network (Atlantic Dialysis Management Services, NY, USA). The Atlantic Dialysis Quality and Support Services and Research Department provided ethical approval and oversight, and the 13 respective participating dialysis clinics' governing bodies also approved this retrospective data analysis. Patient consent was not required since this was a retrospective assessment of a quality improvement project. Additionally, all patient data was de-identified, and confidentiality of data was maintained throughout the course of the study.

Patients dialyzed via CVCs represent approximately $19 \%$ of the 2400 patients served by the clinics. CVC use and CLABSI incidence data from a 5-month period (May to September 2018) was used for an initial assessment. Given the substantial CLABSI rate reduction with the group who received hemodialysis with chlorhexidine-coated end caps ("chlorhexidine group"), almost all patients were switched to the chlorhexidine-coated end caps by February 2019 and follow-up data collection concluded in June 2019.

\section{Materials and Procedures}

The chlorhexidine-coated CVC caps used were ClearGuard HD Antimicrobial Barrier Caps (ICU Medical, San Clemente, CA, USA), which are equipped with chlorhexidine-coated cap threads and a chlorhexidine-coated rod that extends into the hemodialysis catheter hub (Figure 1). Therefore, attaching the cap to the dialysis catheter hub results in the local dissolution of concentrated chlorhexidine within the lock solution between the tubing clamp and the cap. The chlorhexidine-coated CVC cap was removed from the catheter at the time of dialysis and discarded. The dialysis catheter hub was then cleaned with an alcohol wipe. The lock solution was aspirated until $5 \mathrm{~mL}$ of

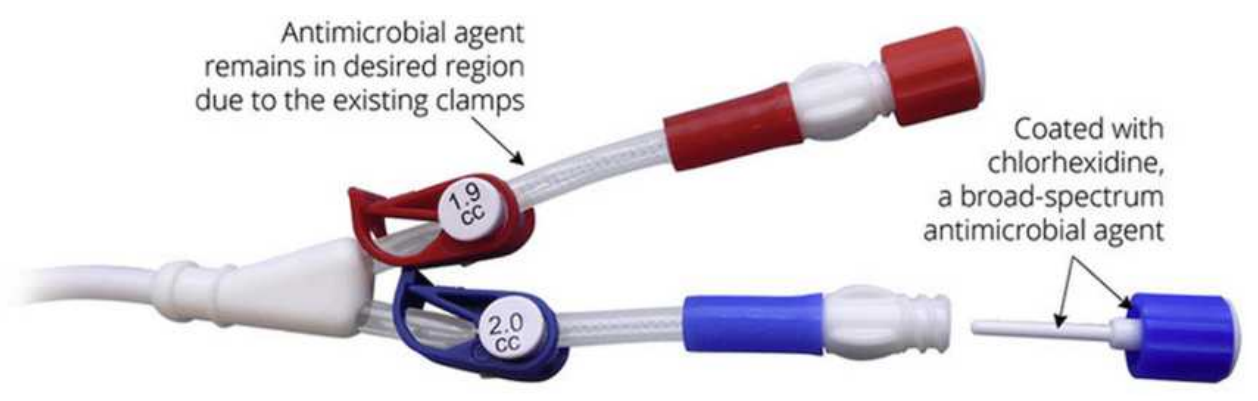

Figure I Antimicrobial barrier cap (ClearGuard $\left.{ }^{\mathrm{TM}} \mathrm{HD}\right)$ used in the chlorhexidine group. 
blood returned into the syringe, and dialysis was initiated. At the completion of dialysis, the catheter was flushed and locked with saline, the hub was cleaned with an alcohol wipe, and a new chlorhexidinecoated CVC cap was applied.

The standard end cap group ("standard group") underwent hemodialysis with the components and procedures previously used at the dialysis network. The catheters were capped by needlefree connectors (Tego, ICU Medical, San Clemente, CA, USA), which remained on the catheter during and after dialysis. The aspiration, flush and locking procedures were identical to those in the chlorhexidine group. However, in the standard group, the connector itself was not additionally capped and was typically discarded and replaced after three dialysis sessions. At the time of connector replacement, the CVC hub was also scrubbed with commercial disinfectant (Alcavis 50, Alcavis Intl., Gaithersburg, MD, USA).

\section{Analysis}

CVC patients and days per study group were estimated from the total number of CVC patients per month. CLABSI counts were recorded by month, and rates were reported as CLABSI/ 1000 CVC days. Chi-squared tests assessed significance of CLABSI/1000 CVC days between the chlorhexidine and standard groups. Data were collected with Microsoft Excel (Microsoft Corporation, Redmond, WA, USA) and statistical analyses were performed using SAS 9.4 (SAS Institute, Cary, $\mathrm{NC}, \mathrm{USA})$.

\section{Results}

Across 13 dialysis centers, de-identified health records of 5934 patients who were dialyzed via CVCs between May 2018 and June 2019 were analyzed. The mean age was 61.3 and $47.1 \%$ of all patients were female. In the first 5-month study period, 967 patients and 1044 patients were identified in the chlorhexidine and standard groups, respectively. One patient was excluded based on physical incompatibility of CVC with chlorhexidine-coated CVC caps, and no patients were excluded based upon allergy-informed contraindications to chlorhexidine. CLABSI rates during the first study period (Table 1) were significantly lower in the chlorhexidine group $(0.03, \mathrm{p}<0.0001)$ relative to the standard group (0.70). The second study period included 3647 and 276 patients in the chlorhexidine and standard groups, respectively. Monthly CLABSI rates remained low in the chlorhexidine group despite increasing $\mathrm{CVC}$ days due to conversion (Figure 2, Table 2). Combined results (both study periods) showed CLABSI rates of 0.63 in the standard group and $0.09(\mathrm{p}<0.0001)$ in the chlorhexidine group (Table 1). Additionally, no increase in thrombosis was reported in clinics converting to the chlorhexidine-coated CVC caps while using saline as the standard locking solution.

A total of 38 CLABSIs with 42 isolates were identified in this analysis: 34 CLABSIs were monomicrobial and 4 CLABSIs were polymicrobial (Table 3). Out of the 42 isolates, $59.5 \%$ were gram-positive organisms, the most common of which were coagulase-negative staphylococci (26.2\%, $\mathrm{n}=11)$, followed by methicillin-resistant Staphylococcus aureus $(11.9 \%, \mathrm{n}=5)$. Gram-negative organisms accounted for $40.5 \%$ of all identified isolates, with Acinetobacter baumannii being the most common organism in this group $(9.5 \%, n=4)$.

\section{Discussion}

Prior to the two study periods used for this analysis, the CVC CLABSI rate across all 13 dialysis network clinics paralleled the national average for outpatient dialysis facilities. ${ }^{5}$ After period one, dialysis clinics utilizing chlorhexidine-coated CVC caps reported significantly lower CLABSI rates, while clinics using standard needlefree connectors continued

Table I Comparison of CLABSI Rates by Study Group

\begin{tabular}{|c|c|c|c|c|c|}
\hline Study Group & Total Number of Patients (N) & CVC Days & CLABSI & CLABSI/I000 CVC Days & $p$-value \\
\hline \multicolumn{6}{|c|}{ First Study Period } \\
\hline Chlorhexidine & 967 & 29,010 & 1 & 0.03 & $<0.0001$ \\
\hline Standard Therapy & 1044 & 31,320 & 22 & 0.70 & \\
\hline \multicolumn{6}{|c|}{ First + Second Study Periods } \\
\hline Chlorhexidine & 4614 & 138,420 & 13 & 0.09 & $<0.0001$ \\
\hline Standard Therapy & 1320 & 39,600 & 25 & 0.63 & \\
\hline
\end{tabular}

Abbreviations: CLABSI, central line-associated bloodstream infection; CVC, central venous catheter. 


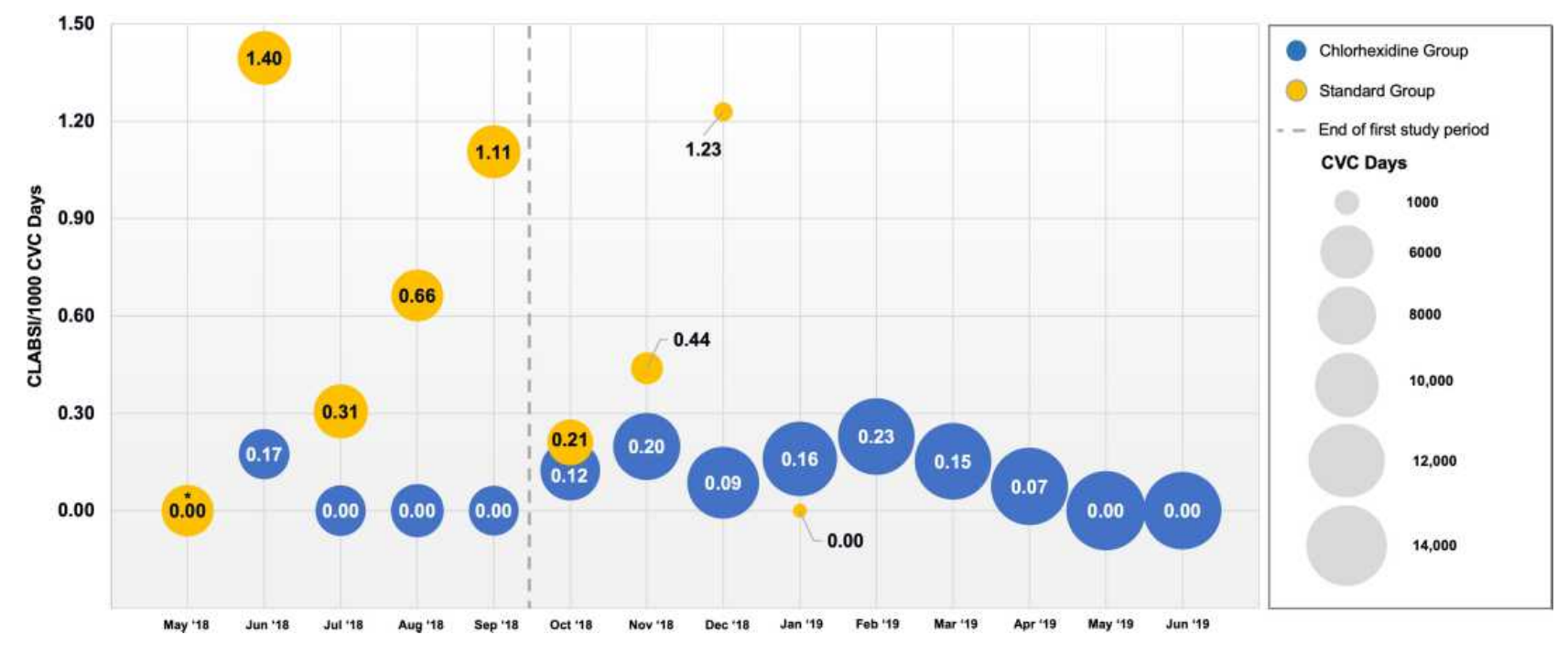

Figure 2 CLABSI/I000 CVC days by study group.

Notes: *Standard group had 0 CLABSIs out of 5970 CVC days and chlorhexidine group had 0 CLABSls out of 5670 CVC days during May 2018.

Abbreviations: CLABSI, central line-associated bloodstream infection; CVC, central venous catheter.

to show rates similar to the national average. ${ }^{5}$ Therefore, the remaining clinics were transitioned to using chlorhexidinecoated CVC caps with minimal disruption to unit workflow and widespread acceptance by clinical staff and patients. Following the second study period, the combined CLABSI rate for the entire 13-clinic network using chlorhexidinecoated CVC caps was 0.09 per thousand CVC days and identical to the NHSN-reported CLABSI rate for hemodialysis via AVFs. ${ }^{5}$

The reduction in CLABSI rates reported from this analysis exceeded the results of previously conducted trials evaluating chlorhexidine-coated CVC caps, ${ }^{9,10}$ and is inclusive of summer months, which have historically demonstrated an upward trend of bacterial infections. ${ }^{11}$

The significant reduction of CLABSI rates is most likely attributable to the chlorhexidine-coated CVC caps, considering no other systemic changes to infection control or CVC care practices were implemented during this period, and comparable systemic practices were utilized for both groups. Additionally, chlorhexidine has been shown to reduce microorganism surface adherence and biofilm formation. ${ }^{12-15}$ Chlorhexidine-coated end caps likely impeded contamination and biofilm formation since attaching the cap to the dialysis catheter hub results in the local dissolution of concentrated chlorhexidine within the lock solution between the tubing clamp and the cap, which is removed prior to dialysis (Figure 1). Comparatively, standard needlefree connectors lack inherent antimicrobial properties and promote safe intravenous access by maintaining a closed lumen when not engaged by mating luers. ${ }^{16}$ The commercial disinfectant used in the standard group to clean the catheter hub was specifically developed for antimicrobial effect in hemodialysis catheter applications and, therefore, unlikely to contribute to the higher rates of CLABSI. ${ }^{17}$

In accordance with the latest Clinical Practice Guidelines for Vascular Access from KDOQI ${ }^{8}$ this study showed that the use of an antimicrobial barrier cap was associated with significant reductions in CLABSI incidence among patients hemodialyzed via CVCs and further suggests that antimicrobial barrier cap use is an effective strategy in preventing CVC dysfunction.

Despite presenting significant reductions in CLABSI rates with the use of chlorhexidine-coated CVC end caps, there are several limitations to the inferences from this study. Since this was a retrospective analysis of a quality improvement initiative, it was not possible to match data from the chlorhexidine and standard groups because detailed patient demographics and medical history were limited. Therefore, direct comparison was not possible, hindering the generalizability of this study's results to a larger outpatient hemodialysis patient population. Multi-center studies with larger and geographically diverse cohorts may provide further evidence regarding the real-world benefits of using chlorhexidine-coated caps in CVC hemodialysis.

\section{Conclusions}

Patients hemodialyzed via CVCs are at increased risk of bloodstream infections. Based on the reduction in CLABSI 


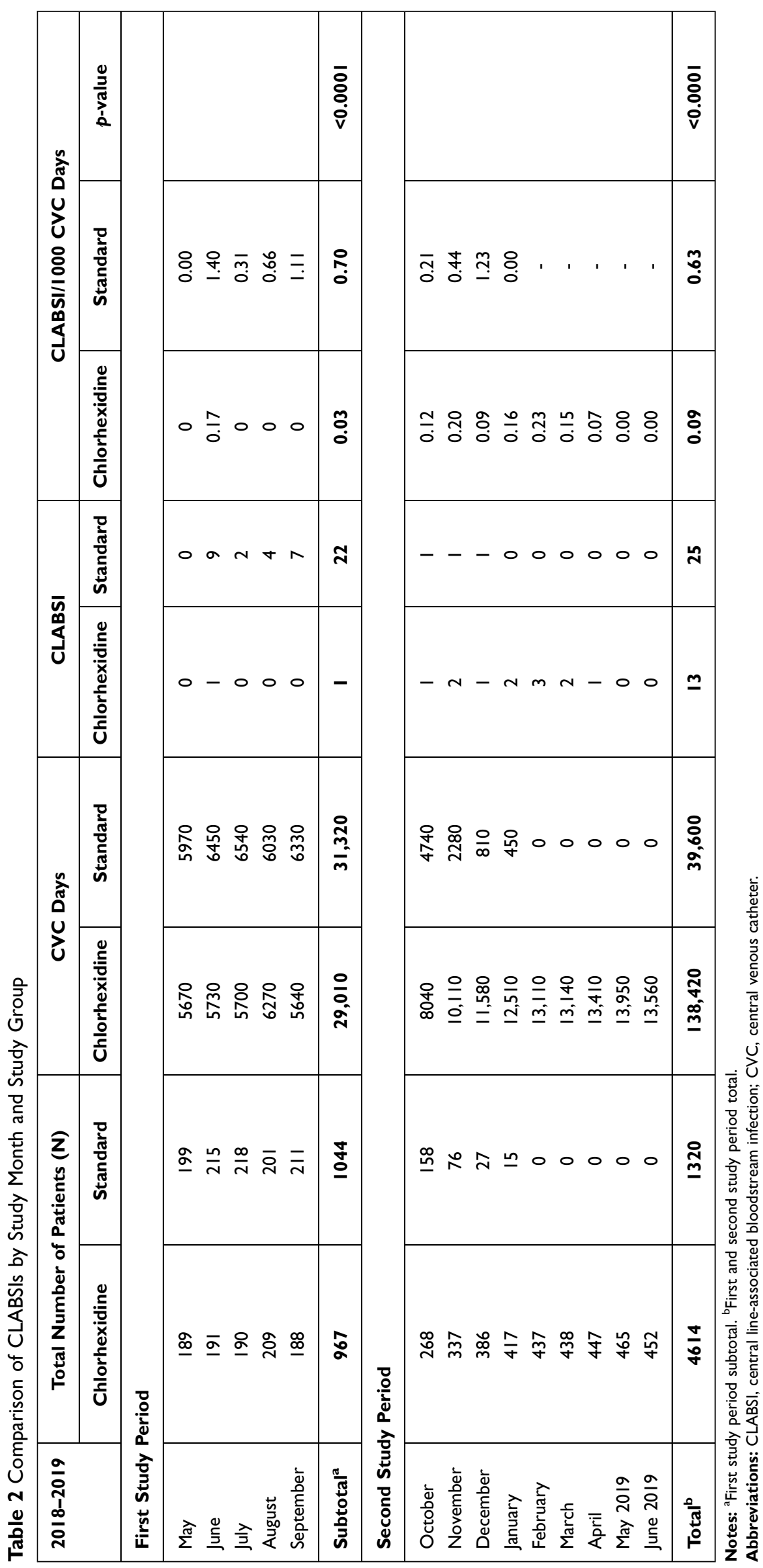


Table 3 Causative Organisms Isolated from Central-Line Associated Bloodstream Infections Among Patients Dialyzed via Central Venous Catheters

\begin{tabular}{|l|c|}
\hline Isolates (N=42) & n (\%) \\
\hline Gram-positive organism & $25(59.5)$ \\
\hline Coagulase-negative Staphylococcus Species (CoNS) & $\mathrm{II}(26.2)$ \\
Methicillin-resistant Staphylococcus aureus (MRSA) & $5(1 \mathrm{I} .9)$ \\
Staphylococcus epidermidis & $3(7.1)$ \\
Staphylococcus aureus & $2(4.8)$ \\
Staphylococcus capitis & $\mathrm{I}(2.4)$ \\
Enterococcus faecalis & $\mathrm{I}(2.4)$ \\
Vancomycin-resistant Enterococcus faecalis & $\mathrm{I}(2.4)$ \\
Streptococcus salivarius & $\mathrm{I}(2.4)$ \\
\hline Gram-negative organism & $\mathrm{I} 7(40.5)$ \\
\hline Acinetobacter baumannii & $4(9.5)$ \\
Entereobacter clocae & $3(7.1)$ \\
Pantoea agglomerans & $2(4.8)$ \\
Escherichia coli & $2(4.8)$ \\
Klebsiella pneumoniae & $2(4.8)$ \\
Serratia marcescens & $\mathrm{I}(2.4)$ \\
Stenotrophomonas maltophilia & $\mathrm{I}(2.4)$ \\
Raoultella planticola & $\mathrm{I}(2.4)$ \\
Citrobacter Freundii & $\mathrm{I}(2.4)$ \\
\hline
\end{tabular}

Notes: 38 central line-associated bloodstream infections (CLABSIs) with 42 isolates were identified among patients dialyzed via central venous catheters; 34 CLABSIs were monomicrobial; 4 CLABSIs were polymicrobial.

rates, findings from this quality improvement report indicate that using chlorhexidine-coated CVC caps may provide a therapeutic improvement. Additional potential benefits of this conversion include improvement in a facility's Quality Incentive Program (QIP) score and reduced costs of treating infections with medications that are not billable outside of the Medicare bundled reimbursement rate. Reduced infection rates also have the potential to decrease hospitalization rates and increase patient satisfaction, both of which may increase unit revenue. Finally, given that every infection carries mortality risk, this analysis suggests that chlorhexidine- coated CVC caps also have the potential to decrease a dialysis unit's mortality rate.

\section{Abbreviations}

AVF, arteriovenous fistulas; AVG, arteriovenous grafts; BSI, bloodstream infection; CLABSI, central lineassociated bloodstream infection; CVC, central venous catheter; KDOQI, Kidney Disease Outcomes Quality Initiative; NHSN, National Healthcare Safety Network; QIP, Quality Incentive Program; VRBSI, vascular accessrelated bloodstream infection.

\section{Ethics Statement}

The Atlantic Dialysis Quality and Support Services and Research Department provided ethical approval and oversight, and the 13 respective participating dialysis clinics' governing bodies also approved this retrospective data analysis. Patient consent was not required since this was a retrospective assessment of a quality improvement project. Additionally, all patient data was de-identified, and confidentiality of data was maintained throughout the course of the study.

\section{Acknowledgments}

The authors would like to acknowledge Atlantic Dialysis Management Services and its affiliates for the support of this study. The authors would also like to acknowledge Dr. Rahul Rajkumar, Dr. Kathryn P. Wall, Dr. Halit O. Yapici, and Dr. Xuan Zhang from Boston Strategic Partners, Inc. (supported by ICU Medical, Inc.) for their statistical analysis and editorial support.

\section{Funding}

Financial support for this study was provided by ICU Medical, Inc, 951 Calle Amanecer, San Clemente, CA 92673 in the form of editorial support and statistical analysis.

\section{Disclosure}

The authors declare no competing financial interests and no conflicts of interest in this work.

\section{References}

1. Viscoli C. Bloodstream infections: the peak of the iceberg. Virulence. 2016;7(3):248-251. doi:10.1080/21505594.2016.1152440

2. Santoro D, Benedetto F, Mondello P, et al. Vascular access for hemodialysis: current perspectives. Int $J$ Nephrol Renovasc Dis. 2014;7:281-294. doi:10.2147/IJNRD.S46643

3. United States Renal Data System. 2018 USRDS annual data report: end-stage renal disease in the United States. National Institutes of Health, National Institute of Diabetes and Digestive and Kidney Diseases. Bethesda, MD; 2018.

4. Zimlichman E, Henderson D, Tamir O, et al. Health care-associated infections. JAMA Intern Med. 2013;173(22):2039. doi:10.1001/ jamainternmed.2013.9763

5. Nguyen DB, Shugart A, Lines C, et al. National Healthcare Safety Network (NHSN) dialysis event surveillance report for 2014. Clin J Am Soc Nephrol. 2017;12(7):1139-1146. doi:10.2215/CJN.11411116

6. Lok CE, Mokrzycki MH. Prevention and management of catheter-related infection in hemodialysis patients. Kidney Int. 2011;79(6):587-598. doi:10.1038/ki.2010.471

7. Bell T, O'Grady NP. Prevention of central line-associated bloodstream infections. Infect Dis Clin North Am. 2017;31(3):551-559. doi:10.1016/j.idc.2017.05.007 
8. Lok CE, Huber TS, Lee T, et al. KDOQI clinical practice guideline for vascular access: 2019 update. Am J Kidney Dis. 2020;75(4 Suppl 2):S1-S164.

9. Brunelli SM, Van Wyck DB, Njord L, Ziebol RJ, Lynch LE, Killion DP. Cluster-randomized trial of devices to prevent catheter-related bloodstream infection. J Am Soc Nephrol. 2018;29 (4):1336-1343. doi:10.1681/ASN.2017080870

10. Hymes JL, Mooney A, Van Zandt C, Lynch L, Ziebol R, Killion D. Dialysis catheter-related bloodstream infections: a clusterrandomized trial of the clearguard hd antimicrobial barrier cap. Am J Kidney Dis. 2017;69(2):220-227. doi:10.1053/j.ajkd.2016.09.014

11. Lok CE, Thumma JR, McCullough KP, et al. Catheter-related infection and septicemia: impact of seasonality and modifiable practices from the DOPPS. Semin Dial. 2014;27(1):72-77. doi:10.1111/ sdi.12141

12. Bakke CK. Clinical and cost effectiveness of guidelines to prevent intravascular catheter-related infections in patients on hemodialysis. Nephrol Nurs J. 2010;37(6):601-615; quiz 616.
13. Denton G Chlorhexidine In: Disinfection, Sterilization and Preservation. 4th. Philadelphia 1991

14. Koburger T, Hubner NO, Braun M, Siebert J, Kramer A. Standardized comparison of antiseptic efficacy of triclosan, PVP-iodine, octenidine dihydrochloride, polyhexanide and chlorhexidine digluconate. J Antimicrob Chemother. 2010;65(8):1712-1719.

15. Kuyyakanond T, Quesnel LB. The mechanism of action of chlorhexidine. FEMS Microbiol Lett. 1992;100(1-3):211-215. doi:10.1111/j.1574-6968.1992.tb05705.x

16. Brunelli SM, Njord L, Hunt AE, Sibbel SP. Use of the Tego needlefree connector is associated with reduced incidence of catheter-related bloodstream infections in hemodialysis patients. Int J Nephrol Renovasc Dis. 2014;7:131-139. doi:10.2147/IJNRD. S59937

17. Alcavis 50 product information; 2002. Available from: http://www. alcavis.com/products/alcavis50.htm. Accessed December 08, 2020.

\section{Publish your work in this journal}

The International Journal of Nephrology and Renovascular Disease is an international, peer-reviewed open-access journal focusing on the pathophysiology of the kidney and vascular supply. Epidemiology, screening, diagnosis, and treatment interventions are covered as well as basic science, biochemical and immunological studies. The manuscript management system is completely online and includes a very quick and fair peer-review system, which is all easy to use. Visit http://www.dovepress.com/testimonials.php to read real quotes from published authors. 